\title{
CASE REPORT: THE USE OF WISC-IV IN ASSESSING INTELLECTUAL FUNCTIONING
}

Dr. Anthony K. Nkyi, Counseling Center, College of Educational Studies, University of Cape Coast, Ghana E-mail:ankyi@ucc.edu.gh

Received: January, 01.2015. Accepted: February, 26.2015.

Original Article

UDK 159.95.072

\begin{abstract}
The purpose of this paper is to provide detailed description of clinical assessment of the Wechsler Intelligence Scale for Children-Fourth Edition (WISC-IV) that can be used by clinicians to assess the level of intelligence of children and adolescents. The WISC-IV is a standardized intelligence test provides essential information and critical clinical insights into a child's cognitive functioning. Literature review and analysis of how to interpret the WISC-IV are described. A psychological report of a 15 year African girl with the use of WISC-IV is reported and clinical impression is given. Information from several sources including interviews with parents, test scores and behavioral observations were used to develop a hypothesis about the child's skills. Her Full Scale IQ of 125 is classified in the superior or higher range of intelligence. Recommendations about how to address problem areas are discussed.
\end{abstract}

Keywords: Wechsler intelligence, Cognitive functioning, Psychological report, Assessment, Children.

\section{INTRODUCTION}

The Wechsler Intelligence Scale for Children - Fourth Edition (WISC-IV) is the latest edition of the Wechsler scale for children 6 to 16 years old (Wechsler, 2003a, 2003b). According to Sattler and Dumont (2008) the WISC-IV has many strengths that include its excellent standardization, good overall psychometric properties, useful diagnostic information, inclusion of Process scores, generally good administration procedures, good manuals and interesting test materials, helpful scoring criteria, usefulness for children with some disabilities and extensive research and clinical literature with prior versions of the test.

\footnotetext{
Corresponding Author

Dr. Anthony K. Nkyi

Counseling Center, College of Educational Studies, University of Cape Coast, Ghana

E-mail:_ankyi@ucc.edu.gh
}

\section{Overview of the WISC-IV}

The first edition, the Wechsler Intelligence Scale for Children (WISC), was published in 1949. as a downward extension of the adult intelligence testcalled the WechslerBellevue Intelligence Scale (Wechsler, 1939). It was followed by the revision in 1974, called the Wechsler Intelligence Scale for Children - Revised (WISC-R). Another revision, the Wechsler Intelligence Scale for ChildrenThird Edition (WISC III) was published in 1991. The staff of PsychCorp prepared the last two revisions, still cited David Wechsler as the author, even after his death in 1982. The test was revised in order to (a) improve its theoretical foundations, (b) improve its psychometric properties, including providing a more recent norms, (c) enhance its clinical utility and increase developmental appropriateness, and (d) increase user friendliness (Sattler and Dumont, 2008). The WISC-IV was standardized on 2200 children ranging in age from 6 to 16 years children with 11 age group were selected to represent children in the United States (Sattler and Dumont, 2008). The one exception was for the Arithmetic subtest, which was standardized on 1100 children. Stratified sampling was used to obtain the demographic characteristics of age, sex, race/ ethnicity, geographic region, and parental education (used as a measure of socioeconomic status). Each age group was made up 100 boys and 100 girls, except in the Arithmetic standardization group, which had 50 boys and 50 girls in each age group from Euro American, African American, Hispanic American, Asian American, and other. The four geographical regions sampled were Northeast, South, Midwest, and West (Sattler and Dumont, 2008).

There are 10 core subtests and 5 supplemental subsets in the WISC-IV (Sattler and Dumont, 2008). Verbal comprehension comprises similarities, vocabulary, and comprehension (three core subtests) and Information 
and Word Reasoning (two supplemental subtests). Perceptual Reasoning comprises block Design, picture Concepts, and Matrix reasoning (three core subtests) and Picture Completion (one supplemental subtest). Working memory comprises Digit Span and Letter Number sequencing (two core subtests) and Arithmetic (one supplemental subtest). Processing speed comprises coding and Symbol Search (two core subtests) and cancellation (one supplement subtest). The WISC-IV yields seven process scores: Block Design No Time Bonus (DSB), Longest Digit Span Forward (LDSF), longest Digit Span Backward (LDSB), Cancellation Random (CAR), and Cancellation Structured (CAS).

The WISC-IV uses standard scores $(M=100, S D=15)$ for the four Indexes and for the Full scale IQ and scaled scores $(M=10$, $\mathrm{SD}=3$ ) for the 15 subtests. The Full Scale IQ is computed by comparing the sum of children in the child's age group. After each subtest is scored, raw score points are summed and converted to scaled scores for the child's own age group (Sattler and Dumont, 2008).

Sattler and Dumont (2008) indicates that the WISC-IV is considered to have outstanding reliability. For example, the four individual composites and the Full Scale have internal consistency reliability coefficients of 0.81 or above over the entire age range covered in the standardization group. Test-retest reliability coefficients indicate that the WISCIV generally provides stable Indexes and Full Scale IQs. The WISC-IV Full scale IQs range from 40 to 160 at all ages of the test, though the range is insufficient for children who are extremely low or extremely high functioning. Environment:

Family Background and Home

Environmental factors that influence children's intellectual developments and functioning in their prenatal and early development include nutrition; family background and home environment; poverty; schooling and cultural variables. These varieties of conditions can be categorized into three main variables, which are; prenatal variable, general birth process variable and Neonatal variable (Sattler, 2008). Prenatal variables are conditions which occur during the period of pregnancy include abnormalities; infections and illnesses in the mother; radiation; prenatal stress; exposure to alcohol, drugs, or environmental toxins; and injury. According to Alcock and Bundy (2001), several types of infections in pregnant woman that can cause severe mental retardation include rubella, syphilis, or cytomegalovirus. Encephalitis, meningitis, cerebral malaria, human immunodeficiency and virus (HIV), and Lyme disease are infectious diseases can interfere with infants' and children's intellectual development.

The maternal use of heroin, cocaine, alcohol, and marijuana are drugs that affect both prenatal and postnatal intellectual development in children (Asanbe and Lockert, 2006; Willford et al., 2006). Family Background and Home Environment Factors associated with family background and home environment are significantly correlate with children's IQs (Lawlor et al., 2006; Lawlor et al., 2006). Persistent poverty has more detrimental effects on children's intelligence test scores and school achievement than does transitory poverty. The cognitive functioning of children living poverty is often diminished because of high rates of maternal prenatal complications, reduced access to health-promoting resources (e.g., doctors, clinics, information), increased exposure to lead, inadequate nutrition, and inadequate homebased cognitive stimulation. These factors may also affect brain development and consequently intellectual development (Noble et al., 2010). The quality of a school plays a minor role in individual differences in IQ. Between $2 \%$ and $10 \%$ of the variance in children's cognitive functioning may be associated with school quality (Bouchard and Segal, 1985). However, there is a high correlation between years of schooling completed and IQ. The evidence suggests, however, that each extra year of education adds 1 to 3.5 points to an individual's expected adult IQ (Bouchard and Segal, 1985; Ceci, 2003). Berry (2001) maintains that intelligence is shaped by the survival skills needed by people in a culture. For instance, well developed visual discrimination and spatial skills are essential in a culture where hunting is emphasized, whereas reading and writing skills are important in a literate society. Acquired knowledge is influenced by the way a culture stores and transmits knowledge and by how individuals in the culture extract information from their environment. Thus, intelligence develops within a cultural context, and cultures differ in how they value different mental abilities (Nisbett, 2010).

The psychological report is a case study that illustrates the application of a recommended practice based on the administration of the WISC-IV to a 15-year old girl whose parents gave the examiner permission to administer the test to her child for educational purposes. The case provides a good example 
of how information from several sourcesincluding interviews with parents, test scores, consultation with the teacher and behavioral observations were used to develop a hypothesis about the child's skills and recommendations about how to address problem areas. Her Full Scale IQ of 125 is classified in the superior or higher range of intelligence. The names in the study are factitious for confidentiality and pedagogical reasons. The interpretive report for the WISC-IV is designed for use in educational evaluation and counseling for graduate students. Readers should note that no referral or placement should be made solely on the basis of the WISC-IV report without confirmation from other sources.

\section{MATERIALS AND METHODS}

The materials used for the assessment include the 10 core subtests and 5 supplemental subsets in the WISC-IV are made up of the: Comprehension Index (VCI) - measures verbal concept formation, verbal reasoning, and knowledge acquired from one's environment (Wechsler, 2003b). The subtests include: similarities subtest measures verbal concept formation and reasoning. The Similarities subtest requires the child to describe how two objects or Concepts are similar (Chen, $\mathrm{H}$. et al., 2009). Compared to other verbal comprehension subtests such as Vocabulary and Information, this subtest appears to require relatively more inductive reasoning (Chen, $\mathrm{H}$. et al., 2009).

Vocabulary subtest measures word knowledge and verbal concept formation. For picture items the child named the object presented visually. For verbal items, the child defined words that are presented visually and orally. Comprehension subtest measures practical reasoning and judgment in social situations. The subtest requires explanation situations, actions and activities and provides one's ability to understand situations and provide answers to specific problems (Sattelr and Dumont, 2008). The child answered questions based on her understanding of the questions. Information subtest measures how one is able to acquire, retain, and retrieve general factual information. The child answered questions that address a broad range of general knowledge topics. Perceptual Reasoning Index (PRI) - measures perceptual and fluid reasoning, spatial processing, and visual motor integration (Wechsler, 2003b). The sub tests include: In the Block Design subtest, child is asked to recreate a design when viewing a model or a picture. It mainly requires visual processing ability, including perceptions of spatial relations and mental manipulations of visual patterns (Chen, H. et al., 2009).

Picture Concept subsets measures abstract categorical reasoning ability. The child is presented with rows of pictures and was to choose one from each row to form a group with a common characteristic. Although mainly requiring inductive ability, it also involves general information or verbal mediation (Chen, H. et al., 2009; see Flanagan and Kaufman, 2004). Matrix Reasoning. This subtest generally requires manipulating abstractions, rules, generalizations, and logical relationship (Chen, H. et al., 2009). The child selects the response option that completes the matrix or series (Chen, H. et al., 2009). For the Picture Completion subtest, the child is asked to name or point to the important part missing from a picture within a specified time limit (Chen, H. et al., 2009). This task involved visual processing and general information abilities (Chen, H. et al., 2009; see Flanagan and Kaufman, 2004).

Working Memory Index (WMI) requires working memory processes to manipulate orally presented verbal sequences (Wechsler, 2003b). Or to simply orally presented sequential information. The sub tests include: Digit Span subtest measures working memory, requires repeating a series of digits (Sattelr and Dumont, 2008). For Digit Span Forward, the child is asked to repeat the digits as given (Sattelr and Dumont, 2008). For Digit Span Backward, the client was made to read a sequence of numbers and recalled the numbers in reverse order.

Letter-Number Sequencing subtest measures sequential processing and shortterm auditory memory (Sattelr and Dumont, 2008). The child read a sequence of numbers and letters and recalled the numbers in ascending order and the letters in alphabetical order. Arithmetic is a supplementary Working memory subtest that requires solving simple to complex arithmetical concepts and numerical reasoning (Sattelr and Dumont, 2008). Working within a specified time limit, the child mentally solved a series of arithmetic problems. Processing Speed Index (PSI) requires visual perception and organization, visual scanning, and the ability to use hands and eyes efficiently (Wechsler, 2003b). The attention factor is two minutes.

The sub tests include: coding. This subtest requires children to copy symbols paired 
with simple geometric shapes or numbers within a specified time limit (Chen, $\mathrm{H}$. et al., 2009). Besides measuring the processing speed, successful performance on this task may also indicate recall ability (Chen, H. et al., 2009). The child completed this subtest using a response booklet. In the Symbol Search subtest, the child is required to scan a search group and rapidly indicate whether the target symbol(s) matches any of the symbols in the search group. This task involve both processing speed and the ability to perceive and think with visual stimuli (Chen, H. et al., 2009; see Keith et al., 2006). The child completed this subtest using a response booklet. The Cancellation subtest measured visualperceptual recogntiton and speed of visual processing (Sattelr and Dumont, 2008). The subtest requires scanning both a random and a structured arrangement of pictures, and marked target pictures within a specified time limit. The child completed this subtest using a response booklet, and not on his or her digital device. report:

Analyzing the Psychological Wisc-IV

Identifying data: This section provides traditional identifying data, such as name, date of birth, age, date of evaluation, date of report, and grade. Other information may include home address, phone number, and name of school. (In actual practice, the child's last name would be included in the section of the report (Dumont and Rapport, 2008)

Test (s) administered: This section cites the name(s) of the test(s) administered and the test scores.

Reasons for referral: This section begins the narrative portion of the report and explains the reason for the evaluation. It documents what the examiner perceives as the purpose for the evaluation and who initiated the referral (parent, teacher, court). This section also helps to develop the focus of the recommendations and contains information about the child that is related to the referral question. In this report, it indicates that the child was tested so that the examiner could obtain practice administering the WISC-IV, though in actual practice, children may be referred for different reasons. For example, when their academic performance is below grade level, they are acting out in class, they have sustained a brain injury or they need a reevaluation etc. (Dumont and Rapport, 2008).

Background information: This section was included in the report for educative purpose although it does not need to be included in a WISC-IV practice. This section of the report contains information about the child's developmental, educational, and family history as could be done in actual practice (Dumont and Rapport, 2008).

Behavioral observations: This section describes the child's behavior during the examination (e.g., level of cooperation, attention, frustration), including his or her relationship with the examiner (Dumont and Rapport, 2008).

Assessment results and clinical impressions: This section describes the child's performance on the WISC-IV. Normative data and confidence bands with the full Scale IQ are included (Dumont and Rapport, 2008). This section discusses discrepancies and/or consistency among the child's indexes. The paper also gives the differences between the indexes and individual subtest scores needed to reach the 0.5 and 0.01 levels of significance. This section also describes the child's strengths and weaknesses based on the subtest scores. The percentile ranks for the entire subtest scaled scores are also indicated. The child's perceptual reasoning abilities also described as average or above average, as noted by his score on the Block Design, Picture Concepts and Matrix Reasoning subtests. Working memory (or auditory short-term memory) is indicated as above/below average or average, as noted by his score on the Digit Span and LetterNumber Sequencing subtests. Her sequential visual processing abilities are described, as noted by her scores on the Coding and Symbol Search subtests.

Recommendation: This section is optional. The author included this portion for educational purposes although it does not need to be included in a WISC-IV practice report.

Summary: This section summarizes the findings and any recommendations made in the body of the report.

\section{RESULTS AND DISCUSSIONS}

\section{Case: Psychological Report}

\section{Client: LAS. Sex: Female \\ Date of birth: $x x / x x / 98$ \\ Age: 15 yrs 4 months \\ School: MC High School.}

TestingDate: 04/07/2013

Examiner: Anthony Nkyi

Preliminary Information: Sources of information used to gather data for this 
evaluation report include a clinical interview with the client and her mother, and the Wechsler Intelligence Scale for ChildrenFourth Edi-tion (WISC-IV).

The WISC-IV is a standardized intelligence test provides essential information and critical clinical insights into a child's cognitive functioning. It also integrates current conceptualization and recent research to provide the most essential information about a child's strengths and weaknesses. The WISC-IV contains 10 core subtests and 5 additional subtests. These are summed to four Indexes (the Verbal Comprehension Index, the Perceptual Reasoning Index, the Working Memory Index and the Processing Speed Index) and one Full Scale IQ (FSIQ) which ranges from lowest 40 to highest 160 points

Two sessions with the client were necessary that include the clinical interview and administration of the WISC-IV lasted three hours and thirty minutes and took place at the client's residence. The parents of LAS gave consent to participate in the evaluation process. The father signed a consent form and the child assented to indicate in what manner the information would be used.

Reason for Referral: Client is a volunteer for the purpose of assisting the examiner obtain practice administering the WISCIV and to provide assessment for educative purposes.

Test Administered: Wechsler Intelligence Scale for Children-Fourth Edition (WISC-IV). (Fig 1, Table A-1)

Verbal comprehension composite Scaled Score

$\begin{array}{ll}\text { Similarities } & 16 \\ \text { Vocabulary } & 13 \\ \text { Comprehension } & 19\end{array}$

Perceptual reasoning composite Block Design 7

Picture Concepts 11

Matrix Reasoning 12

Working memory composite

Scaled Score

Digit Span 13

Letter-Number Sequencing 14

Processing speed

Coding $\quad$ Scaled Score
Symbol Search 14

Composite scores (Fig 2. Table A- 2)

$\begin{array}{lc}\text { Standard Score } & \text { Percentile Rank } \\ \text { VC I } 136 & 99 \% \\ \text { P R I 100 } & 50 \% \\ \text { W MI } 120 & 91 \% \\ \text { P S I 123 } & 94 \% \\ \text { F S IQ } 125 & 95 \%\end{array}$

Background Information: LAS. is a 15 year African American female high school student who lives with her parent in the state of Nebraska. She is a monolingual English speaker.

The client has an unremarkable medical and mental health history. The client's mother reports that LAS has never had significant medical problems or accidents, and had never been seen by a mental health professional.

LAS was born in the state of Nebraska, the oldest and the only female of four siblings in 1993. Although she was born in the state of Nebraska, her parents had already had originally moved from one of the West African French countries to the United States of America. The parents report that LAS grew up healthy and had no problems during her developmental growth. Her mother holds a Master's degree in Management Studies while the father has a Bachelor's degree.

The mother and the father are employed in a Bank and a business Company respectively.

LAShas other three siblings. Nick a seventh grade, Joe a second grade and Joe in a Kindergarten, all attend a Mission School.

The mother reports that LAS was a healthy full term baby with no problems at birth. She started talking at an early age and started kindergarten a few weeks before she turned five years. LASattended kindergarten through her eight grades at elementary school in the state of Nebraska. The client excelled in all her classes throughout her elementary education. As a result of her academic excellence, she was awarded the Honorary Rotary Club award and The Black Catholic Student Scholarships for high school education.

LAS attends MC high school in Nebraska and has good academic record and won the state champion in Speech in Serious prose in 2009. She is on several committees at school. She is an active Catholic Church member and plays leadership roles in her church youth group. She was selected to be part of Youth Leadership program at the Creighton University and had the opportunity 
to visit several business and social locations in Nebraska throughout the 2008-2009.

LAS is a very sociable and likes to spend time with her friends. The client expressed an interest in hobbies that include reading and socializes with friends.

The client would like to do many things when she grows up. She wants to be a pediatrician, bio-engineer, a teacher and a dancer.

Behavioral Observations:

LAS was compliant with the instructions of the WISC-IV administration. At first, she seemed comfortable with the examiner but showed some signs of anxiety as some of the questions appeared harder and difficult. She often asked "Are we still on the difficult stuff" when the questions appear difficult. She worked calmly and quietly and answered most of the questions with much interest. There were two brief interruptions in the administration of the test. One was a visit to the rest room and the other was to the kitchen to drink some wa-ter.

Presentation and Mental Status:

During the session LAS was alert, and oriented to time, place, and person. She presented no unusual behaviors during the sessions. Rapport with the client was easily established and maintained. Her mood was within normal limits. She was dressed appropriately in a short pant and T-shirt. Client appeared well groomed and exhibited no problems with gait. LAS exhibited good use of the English language and understood instructions of varying complexity. She was very verbal and her responses to questions were direct and appropriate with no evidence of any speech or hearing diffi-culties. Her attention to the task at hand and her concentration were appropriate alt-hough she showed some signs of anxiety with some difficult questions.

WISC-IV interpretation Results:

The purpose of the administration of the Wechsler Intelligence Scale for childrenfourth Edition (WISC-IV) to the volunteered client was to assess her overall level of cognitive functioning and to assist the examiner obtain practice administering the WISC-IV

LAS overall intellectual ability is in the superior range. Her Verbal Comprehension ability is in the very superior range; her Working Memory and Processing Speed abilities are in the superior range while Perceptual reasoning is in the average range The Full Scale IQ (FSIQ) is derived from the combination of the ten subtest scores and is considered the most representative estimate of global intellectual functioning (Ref. Table A-2).
There were significant differences among the four indexes that are associated with relatively low scores on the Perceptual reasoning-Block Design. In spite of the differences, the present measure of her overall thinking, reasoning, and intellectual functioning ap-pear to be valid

LAS's performance on the Verbal Comprehension Index suggests that she performs within the very superior range and above those of $99 \%$ of her age group. (VCI $=136 ; 95 \%$ confidence interval=127-141). The Verbal Comprehension Index is designed to measure verbal reasoning and verbal acquired knowledge. This may suggest her ability to apply verbal skills and information to the solution of new problems, process verbal information and ability to think with words are better developed. The scores may also suggest that her Verbal Comprehension skills are better developed than her perceptual reasoning skills and that retrieval of verbal information from long term memory is better developed than nonverbal problem solving.

LAS's performance on the Perceptual reasoning skills indicate that she is in the average range and above those of approximately $50 \%$ of her age group. The perceptual reasoning composite measures nonverbal reasoning skills. Her low score on Block Design may suggest she has poor visual-perceptual reasoning and non-verbal reasoning ability.

LAS's working memory abilities as measured by the Working Memory Index are in the superior range and above $91 \%$ of her age group. Such high score may suggest that she has the ability to sustain attention and has a high long term and short term auditory memory and attention.

LAS scored in the superior range on the Processing Speed Composite. Her score is equal or above $95 \%$ of people in her age. Processing Speed Index measures processing speed skills. Such superior score suggests that LAS has good processing speed and good attention and concentration. Her strength in processing speed indicates that she has the ability to work under time pressure. The significant difference between the Verbal Comprehension and Perceptual reasoning skills may indicate that her Verbal comprehension skills are better developed than perceptual reasoning skills. The results of the score also suggest that the Verbal comprehension and verbal processing are better developed than her working memory and short term auditory memory. The difference between the verbal comprehension and processing speed skills may suggest that 
the verbal processing and long term memory is better developed than short term visual memory (Table A-3).

LAS.'s scores also reflect a number of strengths. For example, one area of strength suggested by the scores was her social judgment and practical reasoning; analogical visual perceptual reasoning, and her ability to reason deductively and the ability to categorize the meaning of individual words. Her high score on Picture comple-tion compared to Block design may suggest that nonspatial perceptual ability is better developed than spatial visualization ability (Table A-4).

Her weakness on block design may not be related to her academic performance but could be likened to her anxiety at the beginning of the test. This is because her overall superior skills (Full Scale IQ=125) indicate that LAShas the ability to perform well at school and at home. Her involvement in church activities as her leadership roles in youth group confirms the elevated score (Ref: Table A-4).

\section{CONCLUSIONS}

Summary:

LAS is a 15 year African American female high school student who lives with her parent in Omaha, Nebraska. Both of her parents migrated from Togo to America. She is a monolingual English speaker.

LAS was born in October 5, 1998 in $\mathrm{Ne}$ braska, the oldest and the only female of four siblings.

LAS has other three siblings. The client has an unremarkable medical and mental health history.

She agreed to participate in this psychological test to assist the examiner for educative purposes. Her scores on the WISC-IV are believed to be valid indication of her present level of intellectual function-ing.

She obtained a WISC-IV Full Scale IQ of 125 in the superior range equal or above $95 \%$ of her peers. LAS overall intellectual ability is in the superior range. Her Verbal Comprehension ability is in the very superior range; her Working Memory and Processing Speed abilities are in the superior range while Per-ceptual reasoning is in the average range

Recommendation:

On the basis of the present evaluation, LASshould be seen as functioning at a superior range. This is not surprise looking at her academic record, her verbal and reasoning abilities

The following specific recommendations may be considered:

1. LAS is encouraged to focus on activities that involve recognition of visual details. This will enable her to gain skills to analyze and synthesize visual-spatial material and visual-motor coordination.

2. LAS will benefit from relaxation techniques to enable her reduce anxiety that can contribute to the ability to work under time pressure and the willingness to guess when uncertain.

3. LAS will need program on motivations to build her selfesteem during frustrations and anxiety. Much emphasis should include building on her strengths.

4. Her learning style appears to be a verbal/auditory learner as she performs best on these type tasks.

The WISC-IV assessment offers stakeholders the opportunity to: (a) identify reading and earning issues early as well as learning disabilities in children; (b) Understand the individual's learning profile and identify gifted children; (c) make appropriate; accommodations and develop learning plans for individual students; and (d) to determine learning processes, which include both strengths and weaknesses and the impact that they might have on individual student performance (Wechsler, 2003a). 
(IJCRSEE) International Journal of Cognitive Research in Science, Engineering and Education Vol. 3, No.1, 2015.

\section{APPENDICES}

Fig. 1. Total raw score to scaled score conversions

TOTAL RAW SCORE TO SCALED SCORE CONVERSIONS

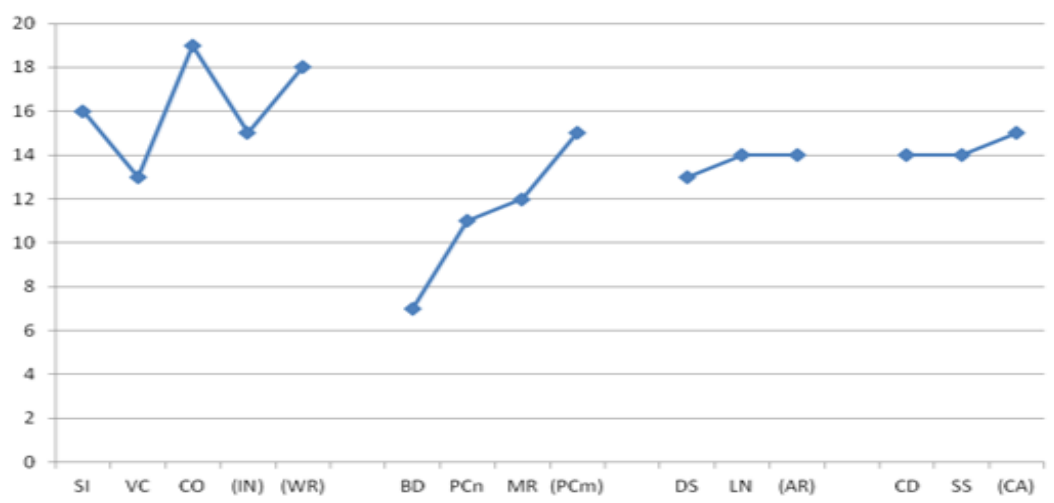

Table A-1.

\begin{tabular}{|c|c|c|c|c|c|c|c|}
\hline Subtest & $\begin{array}{l}\text { Raw } \\
\text { score }\end{array}$ & \multicolumn{6}{|c|}{ Scaled Scores } \\
\hline Block Design & 38 & 7 & & 7 & & & 7 \\
\hline Similarities & 39 & 16 & 16 & & & & 16 \\
\hline Digit Span & 22 & 13 & & & 13 & & 13 \\
\hline Picture Concepts & 21 & 11 & & 11 & & & 11 \\
\hline Coding & 86 & 14 & & & & 14 & 14 \\
\hline Vocabulary & 56 & 13 & 13 & & & & 13 \\
\hline Letter-Number Seq. & 23 & 14 & & & 14 & & 14 \\
\hline Matrix Reasoning & 28 & 12 & & 12 & & & 12 \\
\hline Comprehension & 42 & 19 & 19 & & & & 19 \\
\hline Symbol Search & 42 & 14 & & & & 14 & 14 \\
\hline (Picture completion) & 36 & 15 & & & & & (15) \\
\hline (Cancellation) & 125 & 15 & & (15) & & (15) & (15) \\
\hline (Information) & 30 & 15 & (15) & & & & (15) \\
\hline (Arithmetic) & 31 & 14 & & & (14) & & (14) \\
\hline (Word reasoning) & 24 & 18 & (18) & & & & (18) \\
\hline Sums of Scaled Scores & & & 48 & 30 & 27 & 28 & \\
\hline & & & $\begin{array}{l}\text { Verbal } \\
\text { Comp. }\end{array}$ & $\begin{array}{l}\text { Perc. } \\
\text { Rsng. }\end{array}$ & $\begin{array}{l}\text { Work. } \\
\text { Mem. }\end{array}$ & $\begin{array}{l}\text { Proc. } \\
\text { Speed }\end{array}$ & $\begin{array}{r}\text { Full } \\
\text { Scale }\end{array}$ \\
\hline
\end{tabular}

Fig. 2. Total raw score to scaled score conversions

TOTAL RAW SCORE TO SCALED SCORE CONVERSIONS

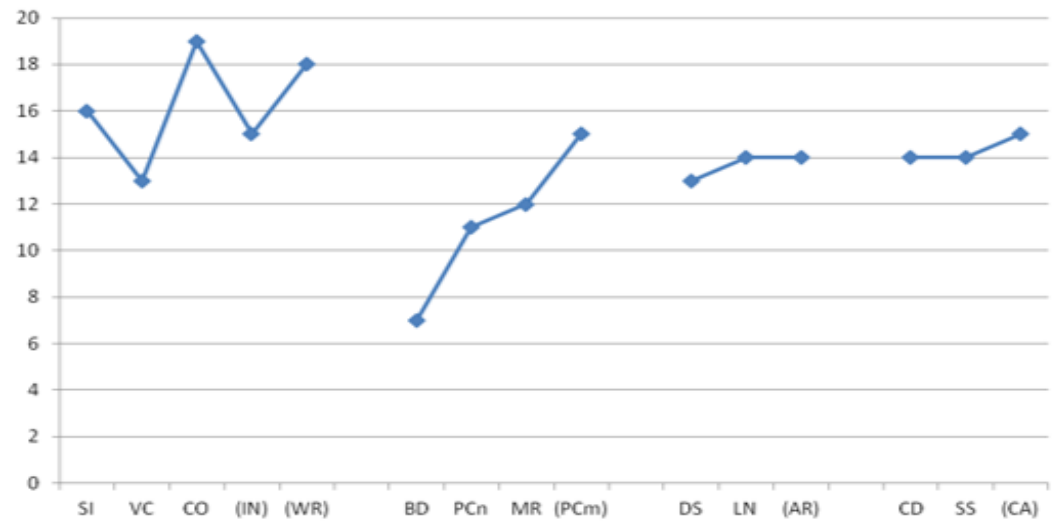


Table A - 2. Sum of scaled scores to composite score conversion

\begin{tabular}{lcccc}
\hline \multicolumn{1}{c}{ SCALE } & $\begin{array}{c}\text { SUM OF } \\
\text { SCALED } \\
\text { SCORES }\end{array}$ & $\begin{array}{c}\text { COMPOSITE } \\
\text { SCORE }\end{array}$ & $\begin{array}{c}\text { PERCENTILE } \\
\text { RANK }\end{array}$ & $\begin{array}{c}\text { 95\% } \\
\text { CONFIDENCE } \\
\text { INTERVAL }\end{array}$ \\
\hline Verbal comprehension & 48 & VCI 136 & 99 & $127-141$ \\
\hline Perceptual Reasoning & 30 & PRI 100 & 50 & $92-108$ \\
\hline Working memory & 27 & WMI 120 & 91 & $111-126$ \\
\hline Processing speed & 28 & PSI 123 & 94 & $111-129$ \\
\hline Full scale & 133 & FSIQ 125 & 95 & $119-129$ \\
\hline
\end{tabular}

Table A-3. Discrepancy comparison

\begin{tabular}{lcccccc}
\hline \multicolumn{1}{c}{ Index/Subtest } & $\begin{array}{c}\text { Scaled } \\
\text { Score 1 }\end{array}$ & $\begin{array}{c}\text { Scaled } \\
\text { Score 2 }\end{array}$ & Difference & $\begin{array}{c}\text { Critical } \\
\text { Value }\end{array}$ & $\begin{array}{c}\text { Significant } \\
\text { Difference } \\
\text { (Y) or (N) }\end{array}$ & Base Rate \\
\hline VCI - PRI & VCI 136 & PRI 100 & 36 & 10.59 & Y & 0.5 \\
\hline VCI -WMI & VCI 136 & WMI 120 & 16 & 10.59 & Y & 20.5 \\
\hline VCI -PSI & VCI 136 & PSI 123 & 13 & 11.38 & Y & 31.7 \\
\hline PRI -WMI & PRI 100 & WMI 120 & -20 & 11.75 & 9.8 \\
\hline PRI - PSI & PRI 100 & PSI 123 & -23 & 12.46 & 3.4 \\
\hline WMI - PSI & WMI 120 & PSI 123 & -3 & 12.46 & 43.9 \\
\hline Digit Span - Letter-N umber Seq. & DS 13 & LN 14 & -1 & 2.08 & 47.1 \\
\hline Coding - Symbol Search & CD 14 & SS 14 & 0 & 2.61 & \\
\hline Similarities - Picture Concepts & SI 16 & PCn 11 & 5 & 2.47 & & 7.7 \\
\hline Stititil
\end{tabular}

Statistical sig. Level- 05

Table A-4. Determining strengths and weaknesses

\begin{tabular}{lcccccc}
\hline \multicolumn{1}{c}{ Subtest } & $\begin{array}{c}\text { Subtest } \\
\text { Scaled } \\
\text { Score }\end{array}$ & $\begin{array}{c}\text { Mean Scaled } \\
\text { Score }\end{array}$ & $\begin{array}{c}\text { Difference } \\
\text { from Mean }\end{array}$ & $\begin{array}{c}\text { Critical } \\
\text { Value }\end{array}$ & $\begin{array}{c}\text { Strength or } \\
\text { Weakness } \\
\text { (S) or }(\mathbf{W})\end{array}$ & $\begin{array}{c}\text { Base } \\
\text { Rate }\end{array}$ \\
\hline Block Design & 7 & 10 & -3 & 3.01 & & 4.20 \\
\hline Similarities & 16 & 10 & 6 & 3.01 & S & 3.70 \\
\hline Digit Span & 13 & 10 & 3 & 2.87 & & 4.80 \\
\hline Picture Concepts & 11 & 10 & 1 & 3.39 & & 4.55 \\
\hline Coding & 14 & 10 & 4 & 3.17 & & 4.90 \\
\hline Vocabulary & 13 & 10 & 3 & 2.70 & & 3.70 \\
\hline Letter-Number Seq. & 14 & 10 & 4 & 2.63 & $\mathrm{~S}$ & 4.50 \\
\hline Matrix Reasoning & 12 & 10 & 2 & 2.68 & & 4.10 \\
\hline Comprehension & 19 & 10 & 9 & 3.44 & $\mathrm{~S}$ & 4.10 \\
\hline Symbol Search & 14 & 10 & 4 & 3.56 & & 4.40 \\
\hline
\end{tabular}

Statistical sig. Level- .05 
(IJCRSEE) International Journal of Cognitive Research in Science, Engineering and Education

Vol. 3, No.1, 2015.

\section{Conflict of interests}

Author declare no conflict of interest.

\section{REFERENCES}

Alcock, K. J., \& Bundy, D. A. P. (2001). The impact of infectious disease on cognitive. In Sternberg, R. J., \& Grigorenko, E. L. (Eds.). (2014). Environmental effects on cognitive abilities. Psychology Press. (pp.221-253). Mahwah, NJ: Erlbaum.

Asanbe, C. B., \& Lockert, E. (2006). Cognitive abilities of African American children with prenatal cocaine/polydrug exposure. Journal of health care for the poor and underserved, 17(2), 400-412.

Berry, J. W. (2001). Contextual studies of cognitive adaptation. Intelligence and personality: Bridging the gap in theory and measurement, 319-333.

Bouchard, T. J., \& Segal, N. L. (1985). Environment and IQ. Handbook of intelligence: Theories, measurements, and applications, 391-464.

Ceci, S. J. (2003). Cast in six ponds and you'll reel in something: looking back on 25 years of research. American Psychologist, 58(11), 855.

Chen, H., Keith, T., Chen, Y., \& Chang, B. (2009). What does the WISC-IV measure? Validation of the scoring and CHC-based interpretative approaches. Journal of Research in Education Sciences, 54(3), 85-108.

Dumont, R., \& Rapport, L. J. (2008). Interpreting the WISC-IV in Sattler, J. M. (2008). Assessment of children: Cognitive foundations. JM Sattler. La Messa, CA: Jerome M. Sattler, Publisher, Inc.

Flanagan, D. P., \& Kaufman, A. S. (2004). Essentials of WISC-IV assessment (Vol. 46). John Wiley \& Sons.

Keith, T. Z., Fine, J. G., Taub, G. E., Reynolds, M. R., \& Kranzler, J. H. (2006). Higher order, multisample, confirmatory factor analysis of the Wechsler Intelligence Scale for Children-Fourth Edition: What does it measure. School Psychology Review, 35(1), 108-127.

Lawlor, D. A., Najman, J. M., Batty, G. D., O'Callaghan, M. J., Williams, G. M., \& Bor, W. (2006). Early life predictors of childhood intelligence: findings from the Mater University study of pregnancy and its outcomes. Paediatric and perinatal epidemiology, 20(2), 148-162.

Nisbett, R. (2010). The Geography of Thought: How Asians and Westerners Think Differently... and. Simon and Schuster.

Noble, K. G., Norman, M. F., \& Farah, M. J. (2005). Neurocognitive correlates of socioeconomic status in kindergarten children. Developmental science, 8(1), 74-87.

Sattler, J. M. (2008). Assessment of children. Cognitive foundations, 5th ed. JM Sattler, San Diego.
Sattler, M. J \& Dumont, R. (2008). WISC-IV Subtests. In Sattler, J. M. (2008). Assessment of children. Cognitive foundations, 5th ed. JM Sattler, San Diego. JM Sattler. La Messa, CA: Jerome M. Sattler, Publisher, Inc.

Wechsler, D (2003a). Wechsler Intelligence Scale for Children-Fourth Edition: Administration and scoring manual. San Antonio, TX: The Psychological Corporation.

Wechsler, D (2003b). Wechsler Intelligence Scale for Children-Fourth Edition: Technical and interpretive manual. San Antonio, TX: The Psychological Corporation.

Wechsler, D. (1939). The measurement of adult intelligence. MD: Williams \& Wilkins.

Willford, J. A., Leech, S. L., \& Day, N. L. (2006). Moderate prenatal alcohol exposure and cognitive status of children at age 10. Alcoholism: Clinical and Experimental Research, 30(6), 1051-1059. 